\title{
Broadband UHF-RFID Passive Tag based on Split- Ring Resonator (SRR) and T-Match Network
}

\author{
Gerard Zamora, Member, IEEE, Simone Zuffanelli, Member, IEEE, Pau Aguilà, Student Member, IEEE, \\ Ferran Paredes, Member, IEEE, Ferran Martín, Fellow, IEEE, and Jordi Bonache, Member, IEEE
}

\begin{abstract}
A novel broadband design of a planar passive UHFRFID tag based on a split-ring resonator (SRR) antenna and the $T$-match network is presented in this work. The radiation properties of the SRR working at its second resonance are exploited to design an RFID tag for the first time. The potential usefulness of the T-match based design methodology to achieve perfect matching between the SRR antenna and any typical RFID chip is demonstrated. A $0.23 \lambda_{0} \times 0.23 \lambda_{0}$ tag has been fabricated. The measured read range is higher than $13 \mathrm{~m}$ within the whole UHF-RFID band with a peak value of $16 \mathrm{~m}$ at $915 \mathrm{MHz}$.
\end{abstract}

Index Terms - Split-ring resonators (SRRs), radiofrequency identification (RFID), planar antennas, T-match network.

\section{INTRODUCTION}

$\mathrm{R}$ ADIO frequency identification (RFID) is a wireless communication technology used to uniquely identify tagged objects, animals or people. Specifically, the use of UHF-RFID systems, which include frequencies between 864 and $928 \mathrm{MHz}$ [1], has experienced a rapid increase in the last years. A passive UHF-RFID tag consists of an antenna matched to a chip, which contains the information about the tagged item. UHF-RFID chips exhibit strongly capacitive input impedance and small resistance [2]; the real part ranging from 15 to $50 \Omega$ and the imaginary part from -100 to $-400 \Omega$. In order to reduce tag cost and size, direct impedance matching techniques are usually preferred over adding an external matching network. However, it commonly requires the use of small antennas $\left(k_{0} a<0.5, k_{0}\right.$ being the resonance wavenumber and $a$ the minimum radius of a sphere enclosing the antenna) that typically exhibit an input resistance lower than the chip resistance. Direct conjugate matching between the antenna and the chip can be achieved by optimizing the antenna geometry [3],[4], by changing the port position [5],[6], or by means of multiple-arm folded dipole configurations [7]. Due to their small size, the resulting tags are intrinsically narrow band. Conversely, broadband tags based on efficient antennas can be designed by properly increasing the antenna size [8]. However, they normally demand the use of matching structures which may degrade tag performance due to additional losses, and produce further increase in tag cost and size. Different

This work was supported by MINECO-Spain (TEC2013-40600-R, TEC2016-75650-R), Generalitat de Catalunya (2014SGR-157), Institució Catalana de Recerca i Estudis Avançats (awarded F. Martín), and by ERDF.

The authors are with GEMMA/CIMITEC (Departament d'Enginyeria Electrònica), Universitat Autònoma de Barcelona. 08193 BELLATERRA (Barcelona). Spain. E-mail: Gerard.Zamora@uab.es. techniques to obtain good impedance matching by adding an external network can be found in the literature [9]-[12]. The most common procedure uses some variant of the T-match network [12]-[15], which adds an inductive component to the antenna input impedance able to compensate the capacitive impedance of the chip. The systematic methodology for designing T-match based UHF-RFID tags proposed in [14] presents three main advantages: (1) the simplicity of applying this method using a resonant antenna and a small structure for the T-match network, which produces an insignificant increase of tag cost and size, (2) the capability of independently control both the real and imaginary parts of the input impedance, maximizing the antenna efficiency $\eta$ at the same time, and (3) the impact of the losses introduced by the T-match network on the antenna gain is negligible, as it will be shown later.

The split-ring resonator (SRR) as a standalone radiating particle has already been used for passive RFID tag design. In [3] and [16], the radiating part is mainly the outer ring, and the inner ring acts as impedance matching. Two near-field tags based on the SRR were presented in [5]. In [4], the range of achievable impedances and radiation efficiencies of the SRR operating as tag antenna was explored, considering different geometrical configurations. The radiation properties of the SRR antenna were studied analytically in [6], and applied to the design of tags in [6], [17] and [18]. In [19], a two turnsspiral resonator was analyzed and a quasi-isotropic tag was proposed. In the abovementioned articles the resonator works close to its fundamental resonance and the resulting tags are only able to operate within a narrow band, mainly due to its small size. In this paper, a new passive UHF-RFID broadband, efficient tag based on an SRR antenna operating at its second resonance, which allows improving the performance, is proposed. Moreover, the potentiality of the T-match based design procedure reported in [14] to achieve perfect matching with any typical RFID chip is demonstrated.

\section{TAG DESIGN}

In order to apply the T-match based tag design methodology presented in [14], a self-resonant antenna operating at $f_{0}$ is required. To this end, the SRR operating at its second resonance is considered in this work. The topology of the SRR is shown in Fig. 1. An analytical expression for the efficiency of the SRR antenna (considering no dielectric) at the second resonance was reported in [20]. Such expression can be rewritten in a simpler form, by fixing the metal conductivity to that of copper $\left(\sigma=5.96 \cdot 10^{7} \mathrm{~S} / \mathrm{m}\right)$, the working frequency 


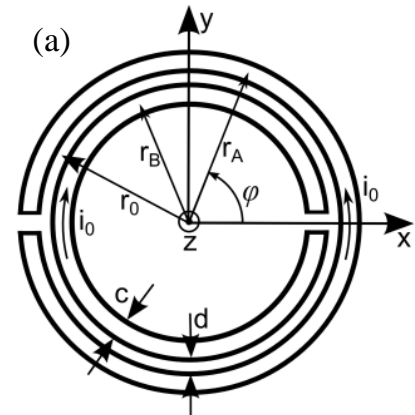

(b)

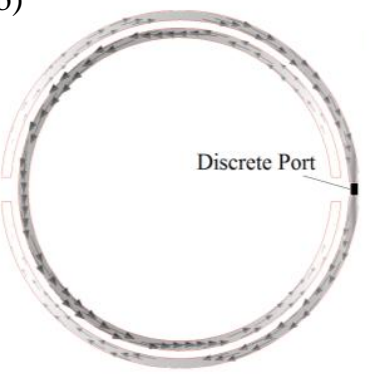

Fig. 1. (a) Sketch of the SRR. (b) Simulated electric current density distribution at the second resonance, $f_{0}$, of the SRR antenna.

$f_{0}=915 \mathrm{MHz}$ (center of the USA band) and considering $h \gg 2 \delta$ ( $h$ and $\delta$ being the conductor thickness and the skin depth, respectively), a condition generally satisfied in the design of planar microwave devices and antennas:

$$
\eta \approx\left[1+\frac{0.94}{c r_{0}}\right]^{-1}
$$

where the dimensions of the conductor width $c$ and the SRR mean radius $r_{0}$ are in $\mathrm{mm}$. Typical values for the mean radius of an SRR operating at its second resonance are in the order of $0.1 \lambda_{0}$ ( $\lambda_{0}$ being the free-space wavelength at $f_{0}$ ). Expression (1) suggests that reasonable values of $c$ provide high antenna radiation efficiencies (e.g., $\eta \approx 98.6 \%$ for $c=2 \mathrm{~mm}$ and $r_{0}=0.1 \lambda_{0}$ ). In order to investigate the usefulness of the T-match based tag design procedure, an SRR antenna working at its second resonance was designed without supporting dielectric substrate. The SRR mean radius was set to $r_{0}=36.2 \mathrm{~mm}$, i.e., approximately $0.1 \lambda_{0}$. The strip width for both rings was chosen to be $c=2 \mathrm{~mm}$. The separation distance between rings was set to $d=1.8 \mathrm{~mm}$ to locate the SRR second resonance at $f_{0}$. An input port was placed at the center of the external ring $\left(\varphi=0^{\circ}\right)$, where a current maximum $i_{0}$ is expected to appear (see Fig. 1a). The simulated input impedance, obtained with the Keysight Momentum software was found to be $70 \Omega$ (similar to half-wave dipoles) at $f_{0}$. The simulated radiation efficiency (98.6\%) perfectly matches with the analytical value obtained from (1). A significant number (33) of RFID tags, achieving conjugate matching between the SRR antenna and 33 different chips were designed following the design steps provided in [14]. The conjugated input impedances seen from the chip were represented with black dots in a Smith Chart (see Fig. 2a). It can be seen that most of the area describing the range of typical chip impedances (delimited by a curved trapezoid) was covered. This demonstrates that the T-match methodology proposed in [14] is useful to achieve perfect matching between the SRR antenna (or any other resonant antenna with similar impedance) and any typical RFID chip. 28 of the designed tags exhibited $\eta>95 \%$, whereas 5 tags reached $90 \% \geq \eta \geq 95 \%$.

The presence of a dielectric substrate is required for tag implementation. It forces a decrease of $r_{0}$ to keep the SRR second resonance at $f_{0}$, giving rise to a reduction of (1). Therefore, a thin substrate with low relative permittivity (typically used in UHF-RFID tag design) should be considered in order to maintain high values of the antenna efficiency. Dielectric losses may also cause a decrease of the radiation
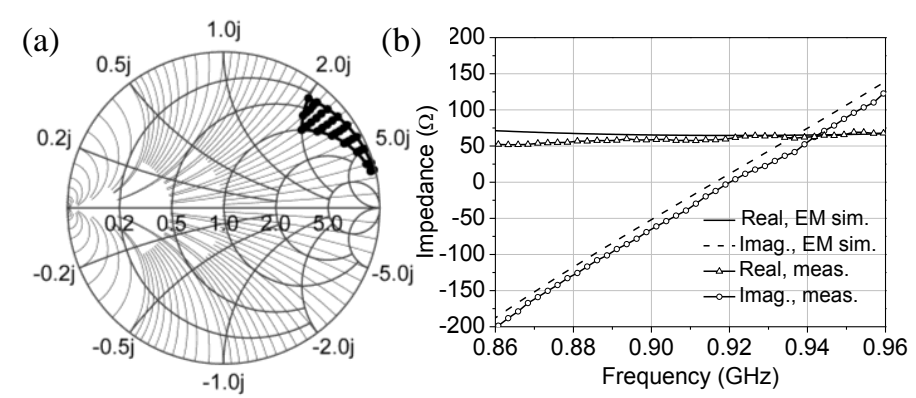

Fig. 2. (a) Conjugated input impedances (normalized to $50 \Omega$ ) of the 33 designed RFID tags (considering no dielectric substrate) in a Smith chart. The typical range of RFID chip input impedances is delimited by a curved trapezoid. (b) Simulated and measured input impedance of the SRR antenna.

efficiency. In practice, however, most dielectric materials have a very small loss, including typical RFID substrates such as PET or paper. In fact, conductor loss is much more significant than dielectric loss at microwave frequencies [21]. Let us now consider a commercial integrated circuit (the Alien Higgs 3 with SOT-323 packaging) for the RFID tag. The input impedance of this chip at the operating frequency $f_{0}$ provided by the manufacturer is $Z_{C}=24.5-j 190 \Omega$. An RFID tag based on an SRR antenna working at its second resonance was designed on a commercial low loss microwave substrate, the Arlon CuClad 250LX with dielectric constant $\varepsilon_{r}=2.43$, loss tangent $\tan \delta=0.0018$, and thickness $h_{s}=0.49 \mathrm{~mm}$. This substrate provides a copper metallization layer of thickness $h=35 \mu \mathrm{m}$. The geometrical parameters of the SRR antenna (see Fig. 1) are $r_{0}=34 \mathrm{~mm}, r_{A}=35.8 \mathrm{~mm}, r_{B}=32.2 \mathrm{~mm}$, $c=2 \mathrm{~mm}$, and $d=1.6 \mathrm{~mm}$. The width of the cuts in each ring was set to $5 \mathrm{~mm}$. The simulated input impedance is shown in Fig. 2(b). The antenna resonance is located at $f_{0}$ and exhibits an input resistance $R_{a}=65 \Omega$. The simulated current distribution of the SRR antenna at $f_{0}$ is depicted in Fig. 1(b). The electric current in the inner and outer rings flows in opposite directions, indicating that the SRR operates at its second resonance [20]. The layout of the designed tag is depicted in the inset of Fig. 3. The strip width of the T-match network (thin loop in Fig. 3) was set to $0.3 \mathrm{~mm}$. According to the matching procedure, the length of the loop was found to be $45.3 \mathrm{~mm}$, and the SRR antenna was connected to the T-match $18 \mathrm{~mm}$ away from the chip position. The simulated power reflection coefficient of the designed RFID tag (see Fig. 3) exhibits perfect matching at $f_{0}$. The simulated radiation pattern is shown in Fig. 4(a). As expected, the radiation pattern in the $E$-plane $\left(\varphi=90^{\circ}\right)$ corresponds to the radiation from an electric dipole oriented towards the $y$-axis. The radiation over the $\mathrm{H}$ plane $\left(\varphi=0^{\circ}\right)$ exhibits a slightly bi-directional pattern, due to the array effect along the $x$-direction associated to the SRR size [20]. Consequently, the simulated directivity of the tag $(2.5 \mathrm{~dB})$ is a bit higher than the value expected from an elemental electric dipole $(1.76 \mathrm{~dB})$. The simulated radiation efficiency of the tag at $f_{0}(\eta=87 \%)$ is slightly lower than the value $(95.7 \%)$ from the simulated SRR antenna.

Cross-polarization of the radiated fields is expected due to the presence of a residual magnetic moment along the axial direction ( $z$-axis). Its maximum contribution is located within the $y z$-plane, more specifically along the $y$-axis $\left(\theta= \pm 90^{\circ}\right)$. In 


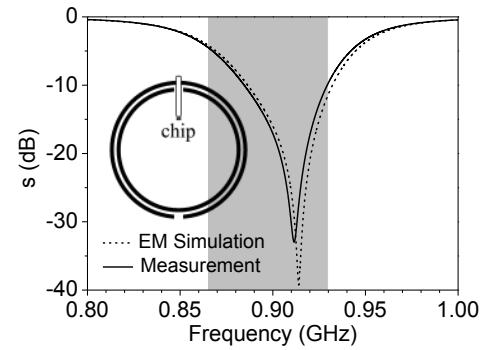

Fig. 3. Simulated and measured power reflection coefficient of the designed tag (inset). The UHF-RFID frequency band is depicted in gray.

such direction, the cross-polarization level (normalized to the maximum value of the co-polar component), assuming no dielectric substrate, can be inferred from [20],

$$
\mathrm{XPOL}_{\max }=9 \pi^{2}\left(\frac{c+d}{\lambda_{0}}\right)^{2} \text {. }
$$

Very low cross-polarization levels $(\mathrm{XPOL}<-21.5 \mathrm{~dB}$ ) were obtained from electromagnetic simulation (see Fig. 4a), which is in very good agreement with the value $\left(\mathrm{XPOL}_{\max }=-20 \mathrm{~dB}\right)$ predicted by (2). Fig. 4(b) shows the simulated axial ratio in the $y z$-plane. It can be observed four regions depicted in gray, where the axial ratio AR remains lower than a certain level, $\mathrm{AR}_{0}=10 \mathrm{~dB}$, which can be considered for elliptical polarization. Since the co-polar radiation is proportional to $\cos ^{2}(\theta)$ and the cross-polar is proportional to $\sin ^{2}(\theta)$, the angle subtended by such regions can be predicted using $\Delta \theta_{\text {ell }}=$ $t g^{-1}\left[\mathrm{AR}_{0} /\left(\mathrm{XPOL}_{\max }\right)^{1 / 2}\right]-\operatorname{tg}^{-1}\left[\mathrm{AR}_{0}{ }^{-1} /\left(\mathrm{XPOL}_{\max }\right)^{1 / 2}\right]$. Each of these regions contain the specific angle where circular polarization $(\mathrm{AR}=0 \mathrm{~dB})$ is achieved, given by $\theta_{c}=$ $\left\{ \pm t^{-1}\left[1 /\left(\mathrm{XPOL}_{\max }\right)^{1 / 2}\right], \pm \operatorname{tg}^{-1}\left[1 /\left(\mathrm{XPOL}_{\max }\right)^{1 / 2}+\pi\right]\right\}$. With the help of (2) it was found that $\Delta \theta_{\mathrm{ell}}=13^{\circ}$ and $\theta_{\mathrm{c}}=\left\{84^{\circ},-84^{\circ}\right.$, $\left.96^{\circ},-96^{\circ}\right\}$, which are in good agreement with the simulation results. It was verified from simulation that, for values of $\mathrm{AR}_{0}$ close to $10 \mathrm{~dB}$, each of the mentioned regions describes approximately a cone in the radiating sphere. Each conical zone subtends a solid angle of $0.04 \mathrm{sr}$. Therefore, the radiated fields of the proposed tag can be considered linear (as commonly desired) on $98.7 \%$ of the radiating sphere.

\section{EXPERIMENTAL RESULTS}

Figure 5(a) shows a photograph of the fabricated tag prototype. The isolated SRR antenna was also fabricated (see Fig. 5b) for comparison purposes, where the T-match network of the proposed tag was replaced by an SMA connector and an SMD packaged balun, to provide differential excitation. The measured input impedance of the SRR antenna is depicted in Fig. 2(b). Good agreement between simulated and experimental results was achieved. The measured radiation pattern is depicted in Fig. 4(a). It can be seen, by comparison with the tag simulation, that the presence of the matching network does not produce a significant variation in the radiation pattern. The simulated tag gain at resonance $\left(G_{r}=1.9 \mathrm{~dB}\right)$ is slightly lower than the measured SRR antenna gain $(2.3 \mathrm{~dB})$. Thus, we can conclude that loss introduced by the T-match network is not significant in terms of gain.

The measured power reflection coefficient of the tag prototype (Fig. 3) was obtained by means of a co-simulation of

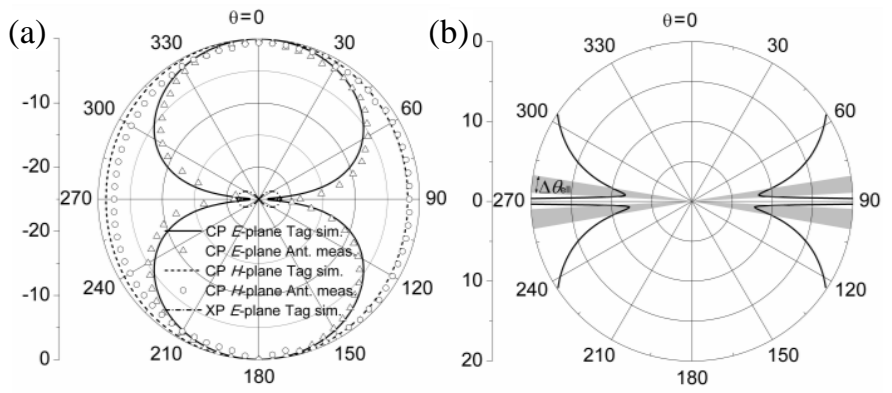

Fig. 4. (a) Radiation pattern (normalized to the simulated tag gain, $\left.G_{r}=1.9 \mathrm{~dB}\right)$ in the $E$-plane $\left(\varphi=90^{\circ}\right)$ and $H$-plane $\left(\varphi=0^{\circ}\right)$ of the simulated Tmatch-based tag, compared to the measured radiation pattern of the fabricated SRR antenna (CP and XP stands for co-polar and cross-polar components, respectively), and (b) simulated axial ratio in the $y z$-plane (regions of low axial ratio $(<10 \mathrm{~dB})$ in gray) of the tag, at $f_{0}=915 \mathrm{MHz}$.

the measured input impedance of the SRR antenna and the simulated T-match network. Simulated and experimental results are in good agreement. Simulated efficiency as well as gain, evaluated at $f_{0}$ and also at both frequency band boundaries, are presented in Table I. A small variation of less than $1 \mathrm{~dB}$ is obtained within the whole UHF-RFID band. The read range of an RFID tag can be calculated as [9]

$$
R R=\frac{\lambda}{4 \pi} \sqrt{\frac{E I R P \cdot G_{r} \cdot \tau}{P_{\text {chip }}}},
$$

where $\lambda$ is the wavelength and EIRP is the equivalent isotropically radiated power, determined by local country regulations (e.g., $3.3 \mathrm{~W}$ in Europe and $4 \mathrm{~W}$ in USA). $P_{\text {chip }}$ is the minimum threshold power necessary to activate the RFID chip (the typical value for the Alien Higgs 3 chip with SOT323 packaging is $-17 \mathrm{dBm}$ ) and $\tau$ is the power transmission coefficient, which is related to the power reflection coefficient $|s|^{2}$ by $\tau=\left(1-|s|^{2}\right)$. The experimental read range was obtained in a TEM cell environment as reported in [6] The tag prototype exhibits a read range (Fig. 6) higher than $13 \mathrm{~m}$ within the whole UHF-RFID band with a peak value of $16 \mathrm{~m}$ at $f_{0}=915 \mathrm{MHz}$. The read range of a commercially available tag (the Alien $A L N-9640$ ) is also shown in Fig. 6 for comparison purposes. This tag is based on a meandered halfwavelength dipole antenna matched to the Alien Higgs 3 chip with no packaging $\left(P_{\text {chip }}=-18 \mathrm{dBm}\right.$, i.e., $1 \mathrm{~dB}$ lower than the chip considered in this work) by means of a T-match network. The length (in the direction of the radiating electric dipole) of the proposed tag $(75 \mathrm{~mm})$ is lower than the length of the Alien $A L N-9640$ tag $(95 \mathrm{~mm})$. Although our fabricated tag has a higher chip threshold power, it exhibits a substantially superior read range within the UHF-RFID frequency band. This is because the presented tag takes advantage of its higher area, producing an array effect that enhances the directivity. Measurements placing the proposed tag over different materials, specifically using $16 \mathrm{~cm} \times 10 \mathrm{~cm} \times 0.2 \mathrm{~cm}$ boards made of metal (with a $2 \mathrm{~cm}$ HDPE foam separation), cardboard paper, wooden, and acrylic plastic, were also carried out (see Fig. 6). These materials shift down the operating frequency band and decrease the read range. However, it is still sufficiently high for many applications. For further investigation, an experimental measurement of the tag prototype in free space was performed in a realistic scenario 

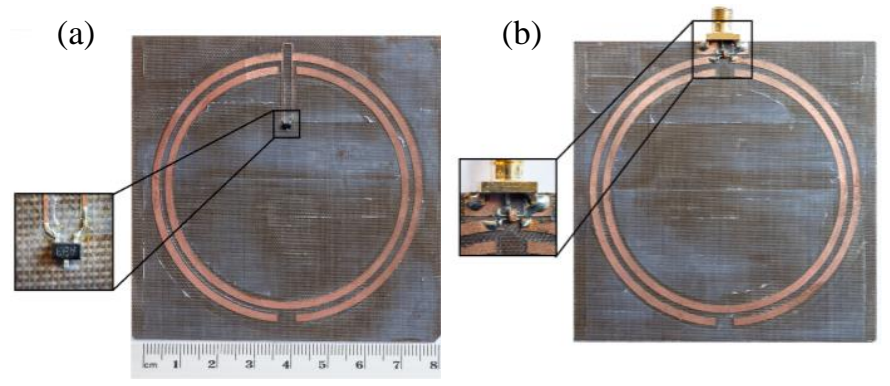

Fig. 5. Photograph of the fabricated (a) tag prototype (the chip is emphasized) and (b) SRR antenna (the SMD-packaged balun is emphasized).

TABLE I

Simulated Gain AND Radiation EFFiciency OF THE PROPOSED TAG

\begin{tabular}{lccc}
\hline \hline Freq.(MHz) & $\mathbf{8 6 4}$ & $\mathbf{9 1 5}$ & $\mathbf{9 2 8}$ \\
\hline Gain $(\mathbf{d B})$ & 1 & 1.9 & 1.7 \\
$\boldsymbol{\eta}(\boldsymbol{\%})$ & 72 & 87 & 84 \\
\hline \hline
\end{tabular}

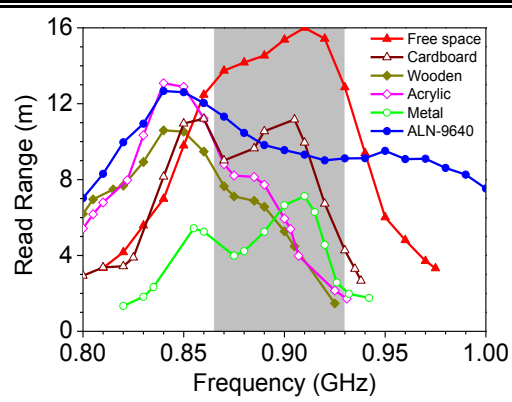

Fig. 6. Experimental read range of the fabricated tag placed over different materials. Comparison with the Alien ALN-9640 tag in free space. The UHF RFID frequency band is depicted in gray.

consisting in a laboratory $(6 \mathrm{~m} \times 6 \mathrm{~m} \times 3 \mathrm{~m}$ height with plasterboard walls and ceiling, and ceramic floor) containing several tags and plenty of furniture, giving rise to multipath propagation. A commercial Motorola FX7400 UHF-RFID reader with an output power of $25 \mathrm{~mW}(14 \mathrm{dBm})$ matched to a half-wave dipole antenna with a gain of $1.64(2.15 \mathrm{dBi})$ was used. The measured read range (normalized to EIRP $=4 \mathrm{~W}$ ) was $13.1 \mathrm{~m}$ and $16.4 \mathrm{~m}$ at the central frequency of the European band $(867 \mathrm{MHz})$ and USA band $(915 \mathrm{MHz})$, respectively. The results are in good agreement with the read range measured using the TEM cell.

\section{CONCLUSION}

In this paper, a new passive UHF-RFID broadband tag based on an SRR antenna, matched to a commercial RFID chip by means of the T-match network, is presented. The SRR antenna was designed to operate at its second resonance to take advantage of its potential radiation properties. Moreover, it has been demonstrated that the T-match network can be used to achieve perfect matching between the SRR antenna (or any other resonant antenna with similar impedance) and any typical RFID chip. Although the size $\left(0.23 \lambda_{0} \times 0.23 \lambda_{0}\right)$ of the proposed tag is higher compared to SRR based tags reported in the literature, the main benefits of using the SRR antenna working at its second resonance are that higher bandwidth, radiation efficiency, and better linear polarization can be achieved. The fabricated tag prototype exhibits dipole-like radiation, linear polarization and a read range higher than $13 \mathrm{~m}$ within the whole UHF-RFID frequency band with a peak value of $16 \mathrm{~m}$ at $915 \mathrm{MHz}$. The results reveal that the proposed tag is very competitive and particularly useful for tagging small items with circular shape.

\section{REFERENCES}

[1] GS1 EPCglobal, Regulatory status for using RFID in the EPC Gen 2 band (860 to $960 \mathrm{MHz}$ ) of the UHF spectrum, 30 Nov. 2016.

[2] E. Bergeret, J. Gaubert, P. Pannier, and J.M. Gaultier, "Modeling and design of CMOS UHF voltage multiplier for RFID in a EEPROM compatible process," IEEE Trans. Circuits Syst. II, Exp. Briefs, vol. 54, no. 10, pp.833-837, Oct. 2007.

[3] T.-H. Cheng, C.-H. Chiang, D.-W. Kung, and S.-Y. Chen, "A compact UHF RFID tag antenna using split-ring-resonator-loaded short dipole," in Proc. APMC, 2014, pp. 453-455.

[4] M. Polivka, A. Holub, M. Vyhnalik, and M. Svanda, "Impedance Properties and Radiation Efficiency of Electrically Small Double and Triple Split-Ring Antennas for UHF RFID Applications," IEEE Antennas Wireless Propag. Lett., vol.12, pp.221-224, 2013.

[5] J. Dong and X. Li, "UHF Near-Field Tags Design Based on Split Ring Resonator", in Proc. APMC, 2011.

[6] S. Zuffanelli, G. Zamora, P. Aguilà, F. Paredes, F. Martín, and J. Bonache, "Analysis of the Split Ring Resonator (SRR) antenna applied to passive UHF-RFID tag design", IEEE Trans. Antennas Propag., vol. 64, no. 3, pp. 856-864, March 2016.

[7] C.A. Balanis, Antenna theory: analysis and design, 3rd ed., John Wiley \& Sons Inc., New Jersey, 2005.

[8] L. J. Chu, "Physical limitations of omni-directional antennas," J. Appl. Phys., vol. 19, pp. 1163-1175, Dec. 1948.

[9] K. V. Seshagiri Rao, P.V. Nikitin, and S.F. Lam, "Antenna design for UHF-RFID tags: a review and a practical application," IEEE Trans. Antennas Propag., vol. 53, no. 12, pp. 3870-3876, Dec. 2005.

[10] G. Marrocco, "The art of UHF RFID antenna design: impedancematching and size-reduction techniques," IEEE Antennas Propag. Mag., vol. 50 (1), pp. 66-79, Feb. 2008.

[11] F. Paredes, G. Zamora, J. Bonache, and F. Martin, "Dual-band impedance-matching networks based on split-ring resonators for applications in RF identification (RFID)," IEEE Trans. Microw. Theory Tech., vol. 58, no. 4, pp. 1159-1166, May 2010.

[12] A. H. Rida, Y. Li, S. Serkan Basat, A. Ferrer-Vidal, S. Nikolaou, and M. M. Tentzeris, "Design, development and integration of novel antennas for miniaturized UHF RFID tags," IEEE Trans. Antennas Propag., vol. 57, no. 11, pp. 3450-3457, Nov. 2009.

[13] D. D. Deavours, "Analysis and design of wideband passive UHF-RFID tags using a circuit model," IEEE Int. Conf. RFID, pp. 283-290, 2009.

[14] G. Zamora, S. Zuffanelli, F. Paredes, F. Martín, and J. Bonache, "Design and synthesis methodology for UHF-RFID tags based on the Tmatch network," IEEE Trans. Microw. Theory Tech., vol. 61, no. 12, pp. 4090-4098, Dec. 2013.

[15] N. A. Mohamed, K. R. Demarest, and D. D. Deavours, "Analysis and synthesis of UHF RFID antennas using the embedded T-match," IEEE Int. Conf. RFID, pp. 230-236, April 2010.

[16] S. H. Lim, Y. C. Oh, H. Lim, Y. S. Lee, and N. H. Myung, "Analysis and design of a UHF RFID tag antenna with a split ring resonator," in Proc. IEEE $i W A T, 2008$, pp. 446-449.

[17] S. Zuffanelli, G. Zamora, P. Aguilà, F. Paredes, F. Martín and J. Bonache, "Passive UHF-RFID tag based on electrically small squareshaped split ring resonator (SRR) antenna," IEEE Int. Symp. Antennas Propag. (APS/URSI), Puerto Rico, USA, 2016.

[18] S. Zuffanelli, G. Zamora, F. Paredes, P. Aguilà, F. Martín, and J. Bonache, "On-metal UHF-RFID passive tags based on complementary split-ring resonators," ," IET Microw. Antennas Propag., vol. 11, no. 7, pp. 1040-1044, 2017.

[19] F. Paredes, S. Zuffanelli, P. Aguilà, G. Zamora, F. Martin, and J. Bonache, "2-SR-based electrically small antenna for RFID applications," Appl. Phys. A, vol.122, no. 4, p. 324, 2016.

[20] S. Zuffanelli, G. Zamora, P. Aguilà, F. Paredes, F. Martín, and J. Bonache, "On the radiation properties of Split Ring Resonators (SRRs) at the second resonance," IEEE Trans. Microw. Theory Tech., vol. 63, no. 7, pp. 2133-2141, July 2015.

[21] D. M. Pozar, Microwave Engineering, 2nd ed. Wiley, NY, 1998. 